\title{
Práticas e saberes em fitoterapia entre os profissionais de saúde de Unidades Básicas de Saúde no Município de Campina Grande, PB
}

\author{
Practices and knowledge in phytotherapy among health professionals in Basic Health Units in the
}

\author{
City of Campina Grande, PB
}

Prácticas y conocimientos en fitoterapia de profesionales de la salud en Unidades Básicas de Salud

de la Ciudad de Campina Grande, PB

Recebido: 11/04/2021 | Revisado: 16/04/2021 | Aceito: 22/04/2021 | Publicado: 03/05/2021

\author{
Nayanne Leal do Monte \\ ORCID: https://orcid.org/0000-0002-2750-4095 \\ Centro Universitário UNIFACISA, Brasil \\ E-mail: nayannelealm@gmail.com \\ Mayse Cristelle de Sales Mélo \\ ORCID: https://orcid.org/0000-0002-9402-0752 \\ Prefeitura do Recife, Brasil \\ E-mail: maysecsm@gmail.com \\ Josefa Raquel Luciano da Silva \\ ORCID: https://orcid.org/0000-0003-1791-5541 \\ Prefeitura do Jaboatão dos Guararapes, Brasil \\ E-mail: jraquel.silva@hotmail.com \\ Rodrigo Pinheiro Fernandes de Queiroga \\ ORCID: https://orcid.org/0000-0002-0642-6382 \\ Universidade Federal de Campina Grande, Brasil \\ E-mail: rodrigopfa@gmail.com \\ Saulo Rios Mariz \\ ORCID: https://orcid.org/0000-0001-7988-9516 \\ Universidade Federal de Campina Grande, Brasil \\ E-mail: sjmariz22@hotmail.com
}

\begin{abstract}
Resumo
Objetivo: Conhecer as práticas e saberes, em fitoterapia, dos profissionais de saúde de Unidades Básicas de Saúde (UBS) no município de Campina Grande, Paraíba. Metodologia: Estudo transversal do tipo exploratório e descritivo, realizado mediante um questionário apropriado. Após a análise estatística descritiva, algumas correlações entre variáveis foram realizadas mediante o teste qui-quadrado e o teste exato de Fischer, com nível de significância de 5\%. Resultados: Entre os profissionais $(n=93)$ pouco mais da metade $(50,6 \%)$ relataram ter adquirido conhecimento sobre fitoterapia por iniciativa própria. Apenas $25,8 \%$ desses profissionais prescreviam ou aconselhavam o uso de plantas medicinais para os pacientes. Em todos esses casos, a relação entre espécie vegetal citada e uso indicado apresentou respaldo na literatura científica; apesar disso, apenas 30 (32,3\%) responderam que se sentem capacitados para prescrever/orientar o uso das plantas medicinais e fitoterápicos. Os entrevistados $(98,9 \%)$ se manifestaram favoráveis à implantação de um programa de fitoterapia na rede municipal de saúde. Como correlações estatisticamente significativas, tivemos que entre os profissionais que possuem um conceito adequado sobre fitoterapia, predominam as mulheres $(\mathrm{p}=0,027)$, e que os profissionais de nível superior prescrevem ou orientam o uso de plantas medicinais e fitoterápicos, mais que os profissionais de nível médio ( $\mathrm{p}=0,047)$. Conclusão: A prescrição e/ou orientação para o uso de plantas medicinais e/ou fitoterápicos não é uma realidade entre a maioria dos entrevistados, sendo necessário investimentos em capacitação desses profissionais de saúde, para a promoção da fitoterapia racional na Atenção Primária em Saúde.
\end{abstract}

Palavras-chave: Fitoterapia; Pessoal de saúde; Atenção primária à saúde; Epidemiologia.

\begin{abstract}
Objective: To know the practices and knowledge, in phytotherapy, of health professionals from Basic Health Units (UBS), in the city of Campina Grande, Paraiba. Methodology: Cross-sectional, exploratory and descriptive study, carried out using an appropriate questionnaire. After the descriptive statistical analysis, some correlations between variables were analyzed using the chi-square test and Fischer's exact test, with a significance level of 5\%. Results and Discussion: Among the professionals $(\mathrm{n}=93)$ just over half $(50.6 \%)$ reported having acquired knowledge about herbal medicine on their own initiative. Only $25.8 \%$ of these professionals prescribed or advised the use of medicinal plants for patients. In all of these cases, the relationship between the plant species mentioned and the indicated use was supported by the scientific literature; despite this, only $30(32.3 \%)$ answered that they feel qualified to prescribe / guide
\end{abstract}


the use of medicinal plants and herbal medicines. Respondents (98.9\%) agree with the implementation of a herbal medicine program in the municipal health system. As statistically significant correlations, we had that among professionals who have an adequate concept about herbal medicine, women predominate $(\mathrm{p}=0.027)$, and that graduated professionals prescribe or guide the use of medicinal plants and herbal medicines, more than non-graduated professionals $(\mathrm{p}=0.047)$. Conclusion: The prescription and / or guidance for the use of medicinal plants and / or phytotherapics is not a reality among most of the interviewees, requiring investments in the training of these health professionals, in order to promote rational phytotherapy in Primary Health Care.

Keywords: Phytotherapy; Healthy personnel; Primary health care; Epidemiology.

\section{Resumen}

Objetivo: Conocer las prácticas y conocimientos, en fitoterapia, de los profesionales de la salud de las Unidades Básicas de Salud (UBS) de la ciudad de Campina Grande, Paraíba. Metodologia: Estudio transversal, exploratorio y descriptivo, realizado mediante un cuestionario adecuado. Después de análisis estadístico descriptivo, se realizaron algunas correlaciones entre variables mediante la prueba de chi-cuadrado y la prueba exacta de Fischer, con un nivel de significancia del 5\%. Resultados: Entre los profesionales $(n=93)$ algo más de la mitad $(50,6 \%)$ refirió haber adquirido conocimientos sobre fitoterapia por iniciativa propia. Solo el $25,8 \%$ de estos profesionales prescribió o aconsejó el uso de plantas medicinales a los pacientes; en todos estos casos, la relación entre las especies vegetales mencionadas y el uso indicado fue apoyada por la literatura científica. Sin enbargo, solo $30(32,3 \%)$ respondieron que se sienten capacitados para prescribir / orientar el uso de plantas medicinales y fitoterápicos. Los encuestados (98,9\%) se mostraron a favor de implementar un programa de fitoterapia en el sistema municipal de salud. Como correlaciones estadísticamente significativas, tenemos que entre los profesionales que tienen un concepto adecuado sobre la fitoterapia, predominan las mujeres $(\mathrm{p}=0,027)$, y que profesionales graduados prescriben o orientan el uso de plantas medicinales y hierbas medicinales, más que los profesionales no graduados $(\mathrm{p}=0,047)$. Conclusión: La prescripción y / o orientación para el uso de plantas medicinales y / o fitoterápicos no es una realidad entre la mayoría de los encuestados, requiriendo inversiones en la formación de estos profesionales de la salud, con el fin de promover la fitoterapia racional en Atención Primaria en salud.

Palabras clave: Fitoterapia; Personal de salud; Atención primaria de salud; Epidemiología.

\section{Introdução}

A utilização de plantas medicinais é uma estratégia de promoção à saúde que vem sendo incorporada ao estilo de vida da população desde a antiguidade, produzindo resultados favoráveis na melhoria da saúde, aumentando a qualidade de vida dos usuários. Além disso, tais produtos possuem menor custo financeiro e maior acessibilidade, trazendo benefícios para quem os utiliza. A implementação do uso das plantas medicinais e fitoterápicos na atenção primária pode se constituir em uma ferramenta bastante eficaz na colaboração com os esforços de concretização dos princípios e objetivos do Sistema Único de Saúde (SUS) na ampliação da assistência integral em saúde (Gontijo \& Nunes, 2017; Lopes et al., 2015).

Muitos estudos demonstram que grande parte da população utiliza esses métodos naturais para o tratamento de diversas patologias, e esse conhecimento empírico sobre a utilidade de cada planta é repassado de geração a geração. No entanto, essa prática não é tão segura quanto o "leigo" pode imaginar e muitas espécies podem apresentar propriedades tóxicas e/ou efeitos adversos. Sendo assim, o emprego terapêutico racional de plantas medicinais se faz necessária. Nesse sentido, é necessário identificar corretamente a planta, sua indicação, dose e forma de preparo corretos, a fim de minimizar os efeitos indesejados (Araújo et al., 2014; Braga \& Silva, 2021; Flor \& Barbosa, 2015; Motta, Lima \& Vale, 2016; Silva \& Barros, 2021; Zeni et al., 2017).

Nesse sentido e para que fosse garantida a integralidade do cuidado, o Ministério da Saúde em 2006 implantou a Política Nacional de Práticas Integrativas e Complementares (PNPIC), tendo como principal objetivo incorporar e implementar as PICS (a homeopatia, as plantas medicinais e fitoterápicos, a medicina tradicional chinesa/acupuntura, a medicina antroposófica e o termalismo social-crenoterapia, entre outras) no SUS, na perspectiva da prevenção de agravos e da promoção e recuperação da saúde, com ênfase na atenção básica, voltada ao cuidado continuado, humanizado e integral em saúde. No mesmo ano foi aprovada a Política Nacional de Plantas Medicinais e Fitoterápicos (PNPMF), estabelecendo diretrizes para a utilização adequada das plantas medicinais e/ou fitoterápicos. As diretrizes apontam fortemente para a estruturação e fortalecimento das cadeias produtivas locais, promovendo assim uma diminuição da dependência estratégica do Brasil, no setor de medicamentos 
fitoterápicos, além de ter como umas das estratégias o estímulo dos profissionais de saúde e da população ao uso racional de plantas medicinais e fitoterápicos. Esses mesmos objetivos, princípios e diretrizes foram mantidos na segunda edição da PNPMF (Brasil, 2016b; Santos et al., 2018; Silva et al., 2021).

Entretanto, para que essas Políticas alcancem o objetivo maior de promover racionalidade terapêutica das PICS, é necessário que os atores envolvidos no processo sejam sensibilizados e capacitados para desempenharem adequadamente seus papéis a fim de viabilizar a implantação de ações concretas que irão, gradativamente, modificar o cotidiano dos serviços. No caso específico da fitoterapia na atenção primária em saúde é fundamental a gestão ter conhecimento dos usuários atendidos pelas Unidades Básicas de Saúde (UBS), que se relacionam com plantas medicinais e fitoterápicos, bem como as práticas e saberes utilizados pela equipe multiprofissional desse nível de atenção em saúde. Assim, os gestores em saúde estarão mais aptos para a elaboração de estratégias e implantação de medidas concretas que visem materializar a PNPMF nas UBS, contribuindo para a promoção de uma fitoterapia racional, o que poderá redundar em melhoria do serviço e aumento da qualidade de vida de cada segmento populacional atendido (Araújo et al., 2015; Feitosa, 2016; Gontijo \& Nunes, 2017; Mariz et al., 2020; Mattos et al., 2018; Nascimento-Júnior et al., 2016; Souza et al., 2016).

Nessa perspectiva, o estudo teve como objetivo fazer um levantamento sobre as práticas e saberes em fitoterapia entre os profissionais de Unidades Básicas de Saúde (UBS) no município de Campina Grande, Paraíba; de forma a analisar o conhecimento científico desses profissionais em relação à fitoterapia, a fim de repassar as informações/indicações corretamente à população para proporcionar ao usuário uma melhoria na qualidade de vida.

\section{Metodologia}

Esse trabalho configurou-se como estudo transversal, do tipo exploratório e descritivo de caráter quantitativo (Marconi \& Lakatos, 2017). Os dados foram coletados no período de janeiro a março de 2019 em 8 (oito) Unidades Básicas de Saúde (UBS), de 7 (sete) diferentes Distritos de Saúde, na Cidade de Campina Grande-PB. Os critérios de inclusão foram: Serem profissionais de saúde (médicos, enfermeiros, técnicos de enfermagem, agentes comunitários de saúde ou odontólogos) atuantes na UBS selecionada, bem como concordar em participar voluntariamente da pesquisa mediante a assinatura do Termo de Consentimento Livre e Esclarecido (TCLE). Sendo assim, os critérios de exclusão foram: Os profissionais de saúde que estivessem afastado do trabalho por algum motivo.

O instrumento de coleta de dados constou de um questionário apropriado (Tomazzoni, 2004) adaptado. Na primeira parte do questionário, havia perguntas sobre o perfil socioeconômico do usuário, com as seguintes variáveis: gênero, idade, bairro, escolaridade e renda familiar que possuíam quando foram entrevistados. A segunda parte contemplava variáveis sobre saberes e práticas dos usuários em fitoterapia. O início da coleta de dados se deu após a aprovação do projeto de pesquisa pelo Comitê de Ética em Pesquisa - CEP do Hospital Universitário Alcides Carneiro - HUAC, em Campina Grande-PB, conforme protocolo CAAE (CEP-HUAC-UFCG) nº 70811717.5.0000.5182.

Foram aplicados um total de 93 questionários nas UBS selecionadas. Os dados foram tabulados em planilha Excel 2016 e analisados mediante distribuição numérica e percentual. Após análise descritiva das variáveis, os dados foram alocados e submetidos à avaliação estatística. As associações entre variáveis foram verificadas através da análise bivariada pelo teste QuiQuadrado ou teste Exato de Fisher. Considerou um nível de 5\% de significância (o mesmo que 95\% de confiança). Um valor de $\mathrm{p}<0,05$ foi considerado para determinar diferença estatisticamente significativa.

Para as análises das associações, as variáveis foram categorizadas em dois grupos, as independentes e as dependentes (Quadro 1). Cada variável independente foi correlacionada com todas as variáveis dependentes. Também foram feitos outros cruzamentos entre os que responderam o conceito de Fitoterapia de forma adequada ou não com os que prescrevem/orientam 
fitoterápicos e plantas medicinais e essa variável com os profissionais que sabem ou não se os pacientes usam as plantas medicinais e/ou fitoterápicos.

Quadro 1 - Variáveis (independentes e dependentes) utilizadas nos testes de associação para avaliar o nível de significância. Campina Grande, 2019.

\begin{tabular}{|c|c|c|}
\hline Variáveis independentes & & Variáveis dependentes \\
\hline $\begin{array}{l}\text { Sexo } \\
\text { Idade } \\
\text { Profissão } \\
\text { Tempo que trabalha na UBS } \\
\text { Renda familiar } \\
\text { Aquisição de conhecimento sobre Fitoterapia }\end{array}$ & $\mathrm{COM}$ & $\begin{array}{l}\text { Prescreve /orienta - Fitoterápicos / Plantas } \\
\text { Medicinais? } \\
\text { Conceito sobre Fitoterapia } \\
\text { Sabe se pacientes usam Plantas Medicinais } \\
\text { e/ou fitoterápicos? } \\
\text { Se sente capacitado para } \\
\text { prescrever/orientar? }\end{array}$ \\
\hline
\end{tabular}

Fonte: Autores (2021).

\section{Resultados e Discussão}

Foram entrevistados 93 profissionais de saúde que atuavam na Atenção Primária em Saúde (APS), distribuídos em 08 Unidades Básicas de Saúde (UBS) do Município de Campina Grande (PB). Em sua maioria, os entrevistados eram: Agentes Comunitários de Saúde (ACS) (50,5\%), do gênero feminino (89,2\%), na faixa-etária de 30 a 39 anos (36,6\%), com renda familiar entre 1 e 2 salários-mínimos (50,5\%) e que já trabalhavam na respectiva UBS por um tempo entre 5 a 10 anos (40,9\%).

Como já era esperado, os ACS foram a categoria profissional predominante. Entretanto, vale ressaltar que a importância desse profissional não se restringe ao aspecto quantitativo, pois são eles os responsáveis pelas visitas domiciliares, em muitos casos pelo primeiro contato de um profissional do serviço com os indivíduos do território. Outra característica dos ACS que merece destaque, principalmente quando se avalia algum aspecto de um serviço em saúde com vistas ao desenvolvimento de estratégias de otimização do mesmo, é que os agentes de saúde possuem maior proximidade, em termos de relações sociais, com os habitantes da área de abrangência da UBS, o que nos deve fazer enxergá-los como elementos essenciais no planejamento de qualquer inovação nos serviços prestados pela unidade de saúde, obviamente, não seria diferente na perspectiva da inserção/ampliação da fitoterapia na atenção primária em saúde. A faixa de renda familiar (em salários-mínimos) predominante apresentou os mesmos valores (numéricos e percentual) das prevalências de ACS na amostra, o que pode nos levar a concluir que existe clara relação entre essas duas variáveis (profissão e renda); ademais, que podemos analisar os dados considerando que eles foram coletados mediantes entrevistas adequadamente conduzidas, gerando confiabilidade dos relatos (Bezerra et al., 2021; Gontijo \& Nunes, 2017; Mattos et al., 2018; Nascimento-Júnior et al., 2016;).

A predominância do gênero feminino em nossa amostra, corrobora como os dados apresentados por outros trabalhos, como os da pesquisa realizada por Nascimento-Júnior et al. (2016), na qual, dentre os 96 entrevistados sobre avaliação do conhecimento e percepção dos profissionais da estratégia de saúde da família sobre fitoterapia em Petrolina-PE, a maioria (n=68, 70,8\%) também era do gênero feminino. Isso mostra, mais uma vez, a forte predominância feminina nas profissões da área da saúde, sobretudo em algumas, como na Enfermagem. No presente trabalho, dos 12 profissionais de Enfermagem com nível superior, todos eram enfermeiras e dos 16 técnicos de enfermagem, apenas 1 era do gênero masculino. No trabalho supracitado, havia apenas 02 enfermeiros entre os 36 entrevistados. Muito tem se discutido sobre tal predominância feminina nas profissões de saúde, que não é um fenômeno apenas brasileiro. Uma das justificativa mais consensuais seria o fato de o cotidiano desses trabalhadores estar intimamente relacionado ao cuidado; como temos uma forte tradição cultural de que às mulheres cabe o papel 
de principais cuidadoras, principalmente no ambiente familiar, elas portanto teriam maior aptidão para a assistência em saúde (Pereira et al., 2021).

A predominância de adultos jovens e a possível constatação de que quase 55\% dos entrevistados possuíam idade entre 30 e 59 anos, nos fazem refletir que os profissionais avaliados correspondem a uma força de trabalho que, provavelmente, ainda estará na ativa minimamente pelos próximos 8 anos. Assim, buscar conhecer potencialidades, interesses e lacunas no conhecimento desses profissionais sobre fitoterapia, subsidiando sólidos investimentos em capacitação profissional com esse público, poderá trazer melhorias para o serviço por um bom tempo. Outra varável analisada na construção desse perfil socioeconômico corrobora essa constatação, é a que evidenciou que a maior parte $(64,5 \%)$ já tem mais de 5 anos no local de trabalho à época, ou seja, muito provavelmente, conhecem bem o serviço e a própria comunidade com a qual trabalham. Portanto, investir em capacitações para este público-alvo seria uma boa estratégia para implantação da fitoterapia racional no serviço de saúde.

Tabela 1 - Distribuição (numérica e percentual) dos entrevistados na pesquisa quanto às variáveis socioeconômicas. Campina Grande, 2019.

\begin{tabular}{|c|c|c|c|c|c|c|}
\hline \multirow{3}{*}{ Variáveis } & \multicolumn{4}{|c|}{ Gênero } & \multicolumn{2}{|c|}{ TOTAL } \\
\hline & \multicolumn{2}{|c|}{ Masculino } & \multicolumn{2}{|c|}{ Feminino } & \multirow[b]{2}{*}{$\mathbf{n}$} & \multirow[b]{2}{*}{$\%$} \\
\hline & $\mathbf{N}$ & $\%$ & $\mathbf{N}$ & $\%$ & & \\
\hline \multicolumn{7}{|l|}{ Faixa etária em anos $(n=93)$} \\
\hline $20-29$ & 1 & 1,1 & 5 & 5,4 & 6 & 6,5 \\
\hline $30-39$ & 1 & 1,1 & 33 & 35,5 & 34 & 36,6 \\
\hline $40-49$ & 4 & 4,3 & 13 & 14,0 & 17 & 18,3 \\
\hline $50-59$ & 3 & 3,2 & 17 & 18,3 & 20 & 21,5 \\
\hline 60 ou mais & 1 & 1,1 & 14 & 15,1 & 15 & 16,1 \\
\hline Ignorado & 0 & 0,0 & 1 & 1,1 & 1 & 1,1 \\
\hline Total & 10 & 10,8 & 83 & 89,2 & 93 & 100 \\
\hline \multirow{2}{*}{\multicolumn{7}{|c|}{$\begin{array}{l}\text { Renda familiar }(n=93) \\
(01 \text { salário-mínimo }=\mathbf{R} \$ 998,00)\end{array}$}} \\
\hline & & & & & & \\
\hline $1-2 S M$ & 5 & 5,4 & 42 & 45,5 & 47 & 50,5 \\
\hline 3-4 SM & 2 & 2,2 & 18 & 19,4 & 20 & 21,5 \\
\hline$\geq 5 S M$ & 3 & 3,2 & 19 & 20,4 & 22 & 23,7 \\
\hline Ignorado & 0 & 0,0 & 4 & 4,3 & 4 & 4,3 \\
\hline Total & 10 & 10,8 & 83 & 89,2 & 93 & 100 \\
\hline \multicolumn{7}{|l|}{ Profissão $(n=93)$} \\
\hline$A C S$ & 7 & 7,5 & 40 & 43,0 & 47 & 50,5 \\
\hline Enfermeiro & 0 & 0,0 & 12 & 12,9 & 12 & 12,9 \\
\hline Médico & 2 & 2,2 & 11 & 11,8 & 13 & 14,0 \\
\hline Odontólogo & 0 & 0,0 & 5 & 5,4 & 5 & 5,4 \\
\hline Técnico de Enfermagem & 1 & 1,1 & 15 & 16,1 & 16 & 17,2 \\
\hline Total & 10 & 10,8 & 83 & 89,2 & 93 & 100 \\
\hline \multicolumn{7}{|l|}{ Tempo de trabalho na UBS $(n=93)$} \\
\hline Menos de 5 anos & 2 & 2.2 & 24 & 25,8 & 26 & 28,0 \\
\hline $5-10$ anos & 5 & 5,4 & 33 & 35,5 & 38 & 40,9 \\
\hline Mais de 10 anos & 3 & 3,2 & 19 & 20,4 & 22 & 23,6 \\
\hline Ignorado & 0 & 0,0 & 7 & 7,5 & 7 & 7,5 \\
\hline Total & 10 & 10,8 & 83 & 89,2 & 93 & 100 \\
\hline
\end{tabular}


Fonte: Autores (2021).

Quando perguntados se os usuários das Unidades de Saúde fazem uso das plantas medicinais e/ou fitoterápicos no tratamento de doenças, mais de $60 \%$ dos entrevistados responderam que "Sim". Esses dados corroboram as informações apresentadas por pesquisas sobre uso de plantas medicinais nos mais diversos segmentos populacionais, inclusive de usuários da atenção primária em saúde, não somente da nossa cidade como também de outras localidades brasileiras. Desse modo, fica claro que que, em sua maioria, os profissionais de saúde têm conhecimento dessa prática por parte dos seus pacientes (Araújo et al., 2014; Braga \& Silva, 2021; Flor \& Barbosa, 2015; Lopes et al., 2015; Mariz et al., 2020; Motta, Lima \& Vale, 2016; Silva \& Barros, 2021; Silva et al., 2018; Silva, Marini \& Melo, 2015; Virginio et al., 2018; Zeni et al., 2017).

Foram citadas pelos profissionais, um total de 166 plantas, como sendo usadas pelos clientes. As mais relatadas corresponderam ao Boldo $(n=29 ; 17,6 \%)$, Cidreira $(n=24 ; 14,5 \%)$, Camomila $(n=21 ; 12,7 \%)$, Hortelã da folha miúda $(n=11$; $6,6 \%)$ entre outras, como Capim- Santo $(n=7 ; 4,2 \%)$, Babosa $(n=4 ; 2,4 \%)$ e Cajueiro roxo $(n=3 ; 1,8 \%)$. As indicações terapêuticas mais citadas foram às doenças do aparelho digestivo (27,9\%), efeito sedativo (23,1\%), doenças do aparelho respiratório $(11,0 \%)$ e doenças do aparelho circulatório $(7.9 \%)$.

Das 166 plantas citadas, com os respectivos relatos de uso terapêutico, apenas 06 não apresentam evidências científicas que respaldem tal uso. São elas: Espinheira Santa para problemas respiratórios, Alecrim para problemas imunológicos, Babosa para doenças respiratórias, Alfazema para doenças oculares e Noni para todos os tipos de doenças. Trabalhos apontam que o Noni possui propriedades anti-inflamatórias, ajuda no combate ao câncer, estimula o sistema imunológico, porém, também pode ocasionar diversos efeitos adversos; portanto, nos pareceu mais adequado classificar o relato de uso do Noni para todos os tipos de doença, como um uso inadequado (Brasil, 2016a; Jahurul et al., 2021). Contudo, o aspecto que merece maior destaque é o fato de que, em quase a totalidade dos relatos, em termos da relação entre planta usada e finalidade terapêutica, existem evidências científicas que corroboram o uso terapêutico da espécie vegetal em questão. Ou seja, os saberes popular e científico não são tão díspares quanto alguns possam imaginar (Flor \& Barbosa, 2015; Mariz et al., 2020).

Durante as entrevistas percebeu-se que alguns profissionais de nível médio ( $\mathrm{n}=10,10,7 \%)$, representados pelos ACS, não apresentaram um conceito sobre fitoterapia, porque não sabiam e desconheciam o termo, sendo explicado seu conceito durante a entrevista. Outros, em proporção semelhante $(n=09,9,7 \%)$, responderam inadequadamente, como "Não sei informar, mas é bastante eficaz", ou ainda: "Bastante relevante". Entretanto, como já era de se esperar, a maioria (n=74, 79,6\%) respondeu adequadamente. Esses percentuais de respostas equivocadas, embora minoritários, ressaltam que uma capacitação sobre Fitoterapia para os profissionais da atenção primária em saúde (APS) não somente é necessária, como urgente.

Quando indagados se achavam a temática relevante na APS, cerca de 89 (95,7\%) eram favoráveis a fitoterapia no SUS; resultado semelhante ao encontrado por estudos prévios (Araújo et al., 2015; Bezerra et al., 2020; Mattos et al., 2018; Nascimento-Júnior, 2016). Em nossa pesquisa, as justificativas apresentadas pelos entrevistados, para tal concordância foram, principalmente: a crença de que as plantas têm poder de cura; que geram menos efeitos colaterais; a possibilidade de uma diminuição no uso de medicamentos industrializados e, ainda, por ser a fitoterapia uma terapêutica acessível e de baixo custo. Outras afirmações relatam a preocupação dos entrevistados quanto às práticas terapêuticas sem embasamento científico e aos desafios da alocação de investimentos necessários para a implementação da fitoterapia no SUS.

Os entrevistados, quando indagados sobre as estratégias de aquisição de informações sobre plantas medicinais e fitoterápicos, destacaram a categoria "leituras e estudos individuais", conforme pode ser visto na Tabela 2. A opção de resposta identificada como "Outros”, com frequências relevantes, representava as informações obtidas por mídias, internet, familiares, amigos e muitas vezes pela própria comunidade em que o profissional atuava, mostrando que a troca de saberes e experiências se faz presente no dia a dia dos que fazem a atenção primária em saúde no nosso município. Ainda sobre essa variável de 
pesquisa, percebe-se que apenas 13,1\% dos profissionais fizeram alguma formação em disciplinas optativas ou obrigatórias durante a vida acadêmica ou participaram de cursos após a graduação. Voltando-se o olhar para a Tabela 1, percebe-se que 32,3\% dos entrevistados eram profissionais de nível superior; ou seja, quase dois terços desses não tiveram contato com fitoterapia ao longo da sua formação profissional. Esse tem sido apontado como um dos principais entraves à implantação da fitoterapia racional na APS. Esses dados, apenas corroboram os resultados de pesquisas semelhantes realizadas em diversas partes do nosso país (Araújo et al., 2015; Bezerra et al., 2020; Feitosa, 2016; Gontijo \& Nunes, 2017; Mattos et al., 2018; Nascimento-Júnior, 2016).

Tabela 2 - Distribuição (numérica e percentual) dos entrevistados, quanto à forma de aquisição de conhecimento em Fitoterapia. Campina Grande, 2019.

\begin{tabular}{ccc}
\multicolumn{2}{c}{ Aquisição de conhecimento sobre Fitoterapia } \\
\hline Desconhece o tema & $\mathbf{N}$ & $(\%)$ \\
\cline { 2 - 3 } Leituras e estudos individuais & 9 & 9,9 \\
Disciplina obrigatória - graduação & 47 & 50,6 \\
Disciplina optativa - graduação & 2 & 2,1 \\
Cursos de curta duração após graduação. & 5 & 5,5 \\
Curso de pós-graduação & 5 & 5,5 \\
Outros & 0 & 0 \\
Total & 25 & 26,4 \\
& 93 & 100 \\
\hline
\end{tabular}

Fonte: Autores (2021).

Em sua maior parte, os profissionais (51,6\%) não prescrevem, nem indicam, plantas medicinais e/ou fitoterápicos para a população atendida na respectiva UBS, porém a maioria admitiu orientar aos usuários quando sabem que eles praticam a fitoterapia $(58,1 \%)$. Todavia, essa informação, na maioria das vezes, não é coletada pelos profissionais, que só ficam sabendo por alguma ocasionalidade. Observou-se, em um estudo qualitativo, que no decorrer das respostas dos usuários houve certa relutância e temor, em informar ao profissional, que estão fazendo uso de algum tipo de chá ou remédio de planta. Os que não falaram, sem que o profissional perguntasse, provavelmente, foi por falta de confiança, por pensarem que vão encontrar rejeição e que seus conhecimentos irão ser vistos como "crendices" (Vargas, 2017). Esse número de não prescrição e/ou indicação de plantas medicinais e fitoterápicos é considerado relativamente alto, quando se pensa no fato de que, em nosso país, existe uma política pública específica nesse sentido; portanto, fica evidente que a ação governamental não deve se restringir a elaborar e aprovar uma determinada política, mas sobretudo, a acompanhar a implementação dessa a fim de identificar as principais dificuldades de concretização, desenvolvendo estratégias para superação de tais empecilhos. Entre as dificuldades para concretização da Política Nacional de Plantas Medicinais e Fitoterápicos, destacam-se: a falta de cursos de aperfeiçoamento sobre o tema, direcionados aos profissionais da APS; pequena quantidade de estudos, bem conduzidos metodologicamente, sobre eficácia e segurança de várias espécies vegetais terapêuticas e, ainda, uma certa resistência dos prescritores para com o uso dos medicamentos fitoterápicos em sua prática clínica (Araújo et al., 2015; Souza et al., 2016).

As plantas citadas pelos profissionais, que responderam que prescreviam e/ou indicavam em fitoterapia, estão descritas na Tabela 3. Todas as indicações terapêuticas para as 124 plantas e os 06 fitoterápicos listados, foram consideradas corretas, conforme a referência bibliográfica citada em cada caso. As plantas mais relatadas foram: Camomila, Boldo, Cidreira e Hortelã; 
assemelhando-se com as mesmas plantas já relatadas, pelos sujeitos de pesquisa, quando questionados sobre quais plantas seus pacientes faziam uso e para qual problema de saúde. Outro aspecto importante desse dado é atentar para o fato de que apenas 01 destas plantas citadas, a Hortelã, está na Relação Nacional de Medicamentos Essenciais (RENAME); um indício de que os profissionais ainda desconhecem as 12 plantas oficialmente listadas na sessão sobre fitoterápicos da edição mais recente de Relação (Brasil, 2020), um documento técnico-científico que deveria orientar a oferta, a prescrição e a dispensação de medicamentos nos serviços do SUS. Outra reflexão que esse dado fomenta é sobre o quanto as ações governamentais na área parecem estar desconectadas do cotidiano de uso popular de plantas medicinais, fato que deveria ser considerado quando das próximas atualizações. Essa situação de desconhecimento das plantas medicinais listadas pela RENAME também já havia sido relatada na pesquisa de Mattos et al. (2018), ao avaliarem 157 profissionais de 66 equipes da Estratégia Saúde da Família (ESF) do município de Blumenau-SC. Os profissionais avaliados nesse estudo, em sua maioria (85,4\%), também desconheciam as espécies vegetais presentes na Relação Nacional de Plantas Medicinais de Interesse do SUS (RENISUS), documento que contém as plantas medicinais que apresentam potencial de avançar etapas da cadeia produtiva e gerar produtos de interesse ao Sistema Único de Saúde.

Ainda na Tabela 3, nota-se a baixa prescrição de medicamentos fitoterápicos, resultando em apenas 06 produtos, sendo somente que 01 deles é disponibilizado no SUS, o Xarope de Guaco. Esse produto só foi citado 03 vezes. Tal fato, associado ao comentário já feito, de que entre as 4 espécies vegetais terapêuticas mais prescritas ou de uso orientado pelos profissionais entrevistados, apenas uma, a Hortelã está na RENAME 2020 (Brasil, 2020), reforça a necessidade de os gestores públicos atuarem no sentido de uma maior capacitação dos profissionais, sobre as potencialidades da fitoterapia na APS no SUS.

Tabela 3 - Distribuição (numérica e percentual) dos entrevistados que prescrevem (e/ou orientam) o uso de plantas medicinais e fitoterápicos, especificando-se o produto, sua indicação terapêutica e referência bibliográfica de suporte. Campina Grande, 2019.

\begin{tabular}{|c|c|c|c|c|c|}
\hline Planta & Nome Científico & Indicação & $\mathbf{n}$ & $(\%)$ & Revisão de Literatura \\
\hline Abacaxi & Ananas comosus & $\begin{array}{l}\text { Doenças do aparelho } \\
\text { respiratório. }\end{array}$ & 3 & $(2,3 \%)$ & Ali et al., 2020 \\
\hline Acerola & $\begin{array}{c}\text { Malpighia } \\
\text { emarginata }\end{array}$ & $\begin{array}{c}\text { Doenças do aparelho } \\
\text { respiratório. }\end{array}$ & 2 & $(1,6 \%)$ & Barros, 2018 \\
\hline Alecrim & $\begin{array}{l}\text { Rosmarinus } \\
\text { officinalis }\end{array}$ & $\begin{array}{c}\text { Doenças do aparelho } \\
\text { digestivo; Sintomas; Efeito } \\
\text { sedativo. }\end{array}$ & 3 & $(2,3 \%)$ & Ladeiras, 2014; \\
\hline Alho & Allium sativum & $\begin{array}{l}\text { Doenças do aparelho } \\
\text { respiratório. }\end{array}$ & 1 & $(0,8 \%)$ & Brasil, 2016a \\
\hline Alpiste & $\begin{array}{c}\text { Phalaris } \\
\text { canariensis }\end{array}$ & $\begin{array}{c}\text { Doenças do aparelho } \\
\text { circulatório. }\end{array}$ & 1 & $(0,8 \%)$ & Valverde et al., 2017 \\
\hline Amoreira preta & Morus nigra & Menopausa. & 4 & $(3,1 \%)$ & Miranda et al., 2020 \\
\hline Babosa & Aloe Vera & $\begin{array}{l}\text { Doenças de pele e do sistema } \\
\text { tegumentar; Cicatrizante. }\end{array}$ & 3 & $(2,3 \%)$ & Brasil, 2016a \\
\hline Boldo & Peumus boldus & $\begin{array}{c}\text { Doenças do aparelho } \\
\text { digestivo; Antidiarreico; } \\
\text { Laxante; Doenças hepáticas. }\end{array}$ & 17 & $(13,2 \%)$ & Brasil, 2016a \\
\hline Cajueiro Roxo & $\begin{array}{l}\text { Anacardium } \\
\text { occidentale }\end{array}$ & Cicatrizante. & 2 & $(1,6 \%)$ & Pirez et al., 2014 \\
\hline Camomila & $\begin{array}{l}\text { Matricaria } \\
\text { chamomilla }\end{array}$ & Efeito sedativo. & 20 & $(15,5 \%)$ & Brasil, 2016a \\
\hline Canela & $\begin{array}{l}\text { Cinnamomum } \\
\text { verum }\end{array}$ & $\begin{array}{c}\text { Doenças do aparelho } \\
\text { digestivo. }\end{array}$ & 1 & $(0,8 \%)$ & Singh et al., 2021 \\
\hline Capim santo & $\begin{array}{c}\text { Cymbopogon } \\
\text { citratus }\end{array}$ & $\begin{array}{c}\text { Doenças do aparelho } \\
\text { digestivo. }\end{array}$ & 6 & $(4,7 \%)$ & Subramaniam et al., 2020 \\
\hline Casca do Maracujá & Passiflora edulis & $\begin{array}{c}\text { Doenças do sistema } \\
\text { endócrino. }\end{array}$ & 1 & $(0,8 \%)$ & He et al., 2020 \\
\hline Cebola branca & Allium сера & $\begin{array}{l}\text { Doenças do aparelho } \\
\text { respiratório. }\end{array}$ & 2 & $(1,6 \%)$ & Kothari et al., 2020 \\
\hline Chá Preto & Camellia sinensis & Doenças da mucosa oral. & 1 & $(0,8 \%)$ & $\begin{array}{c}\text { Tafazoli e Moghadam, } \\
2020 \\
\end{array}$ \\
\hline
\end{tabular}


Research, Society and Development, v. 10, n. 5, e29310514928, 2021

(CC BY 4.0) | ISSN 2525-3409 | DOI: http://dx.doi.org/10.33448/rsd-v10i5.14928

\begin{tabular}{|c|c|c|c|c|c|}
\hline Chuchu & Sechium edule & $\begin{array}{l}\text { Doenças do aparelho } \\
\text { circulatório. }\end{array}$ & 2 & $(1,6 \%)$ & Vieira et al., 2019 \\
\hline Cravo & $\begin{array}{c}\text { Dianthus } \\
\text { caryophyllus }\end{array}$ & Efeito sedativo. & 1 & $(0,8 \%)$ & Chandra et al., 2019 \\
\hline Erva Cidreira & Melissa officinalis & $\begin{array}{c}\text { Doenças do aparelho } \\
\text { digestivo; Antidiarreico; } \\
\text { Efeito sedativo. }\end{array}$ & 11 & $(8,5 \%)$ & Heshmati et al., 2020 \\
\hline Erva doce & $\begin{array}{l}\text { Pimpinella } \\
\text { anisum }\end{array}$ & $\begin{array}{c}\text { Doenças do aparelho } \\
\text { digestivo; Efeito sedativo. }\end{array}$ & 3 & $(2,3 \%)$ & Azimi e Zahedi, 2021 \\
\hline Espinheira santa & $\begin{array}{l}\text { Maytenus } \\
\text { ilicifolia }\end{array}$ & $\begin{array}{l}\text { Doenças do aparelho } \\
\text { digestivo; Doenças do } \\
\text { aparelho respiratório. }\end{array}$ & 5 & $(3,9 \%)$ & Brasil, 2016a \\
\hline $\begin{array}{c}\text { Fitoterápico Camomilina } C \\
\text { (Vitamina C, Extrato de } \\
\text { Camomila, Extrato de Alcaçuz, } \\
\text { Vitamina D3). }\end{array}$ & $\begin{array}{c}\text { Anacardium } \\
\text { occidentale, } \\
\text { Glycyrrhiza glabr. }\end{array}$ & $\begin{array}{l}\text { Sintomas; Doenças do } \\
\text { aparelho digestivo. }\end{array}$ & 2 & $(1,6 \%)$ & Medeiros, 2018 \\
\hline Fitoterápico Arlivry & Hedera helix & $\begin{array}{c}\text { Doenças do aparelho } \\
\text { respiratório. }\end{array}$ & 2 & $(1,6 \%)$ & Barnes et al., 2020 \\
\hline Fitoterápico Pasalix $^{\circledR}$ & $\begin{array}{l}\text { Passiflora } \\
\text { Incarnata } L ., \\
\text { Crataegus } \\
\text { Oxyacantha L., } \\
\text { Salix Alba. }\end{array}$ & Efeito sedativo. & 1 & $(0,8 \%)$ & Silva et al., 2020 \\
\hline Fitoterápico Seakalm & $\begin{array}{c}\text { Passiflora } \\
\text { incarnata L. }\end{array}$ & Efeito sedativo. & 1 & $(0,8 \%)$ & Brasil, 2016a \\
\hline Folha do Abacateiro & Persea americana & $\begin{array}{c}\text { Doenças do aparelho } \\
\text { geniturinário. }\end{array}$ & 1 & $(0,8 \%)$ & Tabeshpour et al., 2017 \\
\hline Folha da Goiabeira & $\begin{array}{c}\text { Psidium guajava } \\
\text { L. } \\
\end{array}$ & Antidiarreico. & 1 & $(0,8 \%)$ & Brasil, 2016a \\
\hline Fitoterápico Xarope Guaco & M. glomerata & $\begin{array}{c}\text { Doenças do aparelho } \\
\text { respiratório. } \\
\end{array}$ & 3 & $(2,3 \%)$ & Brasil 2020 \\
\hline Hibisco & & Emagrecimento; Colesterol. & 2 & $(1,6 \%)$ & Cunha et al., 2016 \\
\hline Hortelã & Mentha & $\begin{array}{l}\text { Doenças do aparelho } \\
\text { respiratório; Doenças do } \\
\text { aparelho digestivo; Doenças } \\
\text { do aparelho circulatório; } \\
\text { Doença parasitária. }\end{array}$ & 9 & $(7,0 \%)$ & Zeljkovi'c ' et al., 2021 \\
\hline $\begin{array}{c}\text { Lambedor } \\
\text { (saião+acerola+alho) }\end{array}$ & $\begin{array}{c}\text { Kalanchoe } \\
\text { brasiliensis, } \\
\text { Malpighia } \\
\text { emarginata, } \\
\text { Allium sativum. }\end{array}$ & $\begin{array}{l}\text { Doenças do aparelho } \\
\text { respiratório. }\end{array}$ & & $(0,8 \%)$ & $\begin{array}{c}\text { Brasil, 2016a } \\
\text { Prakash e Baskaran, } \\
2018 \\
\text { Araújo, } 2020\end{array}$ \\
\hline $\begin{array}{l}\text { Fitoterápico Xarope } \\
7 \text { ervas (Hortelã, Limão, Alho, } \\
\text { Eucalipto, Gengibre, Acerola, } \\
\text { Romã). }\end{array}$ & $\begin{array}{c}\text { Mentha, Citrus limon, } \\
\text { Allium sativum, } \\
\text { Eucalyptus, Zingiber } \\
\text { officinal, Malpighia } \\
\text { emarginata, Punica } \\
\text { granatum. } \\
\end{array}$ & $\begin{array}{c}\text { Doenças do aparelho } \\
\text { respiratório. }\end{array}$ & & $(0,8 \%)$ & $\begin{array}{c}\text { Brasil, 2016a } \\
\text { Prakash e Baskaran, } \\
2018 \\
\text { Dhakad et al., } 2018 \\
\text { Dosoky e Setzer, } 2018 \\
\text { Ge et al., 2021 } \\
\text { Zeljkovi'c' et al., } 2021 \\
\end{array}$ \\
\hline Limão & Citrus limon & $\begin{array}{c}\text { Doenças do aparelho } \\
\text { respiratório. }\end{array}$ & 1 & $(0,8 \%)$ & Dosoky e Setzer, 2018 \\
\hline Limão com alho & $\begin{array}{l}\text { Citrus limon, } \\
\text { Allium sativum }\end{array}$ & $\begin{array}{c}\text { Doenças do aparelho } \\
\text { respiratório. }\end{array}$ & 1 & $(0,8 \%)$ & $\begin{array}{c}\text { Brasil, 2016a } \\
\text { Dosoky e Setzer, } 2018\end{array}$ \\
\hline Louro & Laurus nobilis & $\begin{array}{l}\text { Doenças do aparelho } \\
\text { digestivo. }\end{array}$ & 1 & $(0,8 \%)$ & Caputo et al., 2017 \\
\hline Macela & $\begin{array}{l}\text { Achyrocline } \\
\text { satureioides }\end{array}$ & $\begin{array}{c}\text { Doenças do aparelho } \\
\text { digestivo. }\end{array}$ & 2 & $(1,6 \%)$ & Gomes et al., 2018 \\
\hline Malva Rosa & Alcea rosea & $\begin{array}{c}\text { Doenças do aparelho } \\
\text { respiratório. }\end{array}$ & 1 & $(0,8 \%)$ & Hanif et al., 2019 \\
\hline Maracujá & Passiflora edulis & Efeito sedativo. & 4 & $(3,1 \%)$ & Silva et al., 2020 \\
\hline Moringa & Moringa oleífera & Problemas imonológicos. & 1 & $(0,8 \%)$ & Almeida, 2018 \\
\hline Mastruz & $\begin{array}{c}\text { Dysphania } \\
\text { ambrosioides }\end{array}$ & $\begin{array}{c}\text { Doenças do aparelho } \\
\text { respiratório. }\end{array}$ & 1 & $(0,8 \%)$ & Rida et al., 2019 \\
\hline Melão de São Caetano & Momordica & $\begin{array}{l}\text { Ooenças de pele e do sistema } \\
\text { tegumentar. }\end{array}$ & 1 & $(0,8 \%)$ & Jia et al., 2017 \\
\hline Romã & Punica granatum & Infecções. & 1 & $(0,8 \%)$ & Ge et al., 2021 \\
\hline Tanchagem & Plantago major & Doenças da mucosa oral. & 1 & $(0,8 \%)$ & Adom et al., 2017 \\
\hline Valeriana & $\begin{array}{l}\text { Valeriana } \\
\text { officinalis }\end{array}$ & Efeito sedativo. & 1 & $(0,8 \%)$ & Brasil, 2016a \\
\hline
\end{tabular}


Fonte: Autores (2021).

As indicações mais citadas para o uso das plantas em determinadas patologias foram: doenças do aparelho digestivo (26,2\%), efeito sedativo $(29,5 \%)$, doenças do aparelho respiratório $(16,2 \%)$ e antidiarreico $(4,7 \%)$ (Tabela 3). Em uma perspectiva complementar a essa informação, temos que, segundo o relato dos profissionais entrevistados, os problemas de saúde mais frequentemente tratados com plantas medicinais pelos pacientes que eles atendem, são: doenças do sistema digestório (indigestão, gastrite, gases, vômitos, úlcera, queimação deglutição, diarreia e dispepsia) (30,9\%); estresse, ansiedade e insônia (29,5\%) e doenças do sistema respiratório (asma, bronquite, tosse, gripe, sinusite, pneumonia, expectorante) (16,2\%). Outros 15 (quinze) conjuntos de indicações terapêuticas para as plantas usadas foram mencionados, entretanto, todos com uma frequência de, no máximo, $3 \%$.

A maioria absoluta dos profissionais $(88,3 \%)$ respondeu que participaria de capacitações em Fitoterapia para adquirir mais conhecimentos e repassar orientações para a comunidade, de forma mais eficaz, corroborando os resultados de Mattos et al. (2018). Os que responderam "Não" ou "Talvez", se justificaram como a falta de tempo, uma realidade comum aos profissionais da APS e um desafio permanente em qualquer planejamento de ações com vistas ao aperfeiçoamento e/ou atualização dos profissionais do SUS. Quando perguntados se a Secretária Municipal de Saúde implantasse um programa de Fitoterapia na Atenção Básica, eles indicariam o uso, a maioria (98,9\%) respondeu que "Sim", visto que estariam regulamentados e teriam uma maior confiabilidade nos dados, pois seriam sobre plantas cultivadas na região e consequentemente as mais conhecidas. Esses resultados, analisados em conjunto, nos permitem afirmar que existe uma abertura, entre os profissionais entrevistados, para a implantação de um Programa de Fitoterapia na Atenção Primária em Saúde da Rede SUS e que a oferta de opções de capacitação para esses profissionais, parece ser um dos primeiros passos que devem ser dados objetivando-se a fitoterapia racional.

Ratificando tal necessidade, observou-se que dos 93 entrevistados, apenas 30 (32,3\%) sujeitos de pesquisa responderam que se sentem capacitados para prescrever/orientar o uso das plantas medicinais e fitoterápicos. Sendo eles: 02 médicos (15\%), 07 (58,3\%) enfermeiros e 21 (33,3\%) ACS e Técnicos de Enfermagem. Nenhum, dos 06 odontólogos entrevistados relatou se sentir em condições para prescrever ou indicar tais produtos. Os 63 profissionais que não se sentem capacitados para a prescrição ou indicação, apresentaram justificativas como: "Não tenho conhecimento", "Não obtive orientações nesse aspecto" "Não fiz capacitação específica". Essa mesma situação de pouco atividade em Fitoterapia, entre profissionais da APS no SUS, inclusive com justificativas muito semelhantes, tem sido relatada por outros pesquisas realizadas Brasil afora (Araújo et al., 2015; Bezerra et al., 2021; Mattos et al., 2018; Nascimento-Júnior et al., 2016).

Ainda em relação ao grau de conhecimento dos profissionais entrevistados sobre plantas medicinais, como requisito fundamental para a promoção da fitoterapia racional na APS, questionamos os profissionais de nível fundamental e médio (técnicos de enfermagem e ACS) sobre a possibilidade de as plantas medicinais poderem fazer algum mal à saúde. Supreendentemente, apenas quase a metade (50,8\%) respondeu que "Sim"; sendo, portanto, preocupante a considerável parcela de entrevistados que reproduzem uma espécie de "inconsciente coletivo" de que "se é natural, não faz mal!"; o que se torna algo preocupante, principalmente entre os profissionais de saúde, os quais deveriam atuar como agentes multiplicadores da promoção da fitoterapia racional na atenção básica do SUS. Como todo e qualquer agente que possui substâncias químicas bioativas, as plantas, para terem seu uso medicinal validado, devem ser avaliadas quanto a sua eficácia e segurança terapêuticas. Não devemos considerar apenas que muitas plantas tóxicas são usadas na medicina popular, mas também que a possível toxicidade de plantas medicinais.

Todavia, ao serem questionados se há contraindicações para o uso das plantas medicinais, a ampla maioria $(77,8 \%)$ dos Técnicos de enfermagem e ACS, respondeu que "Sim", e apenas 22,2\%, que "Não", em aparente contradição aos dados 
anteriores; afinal, se há contraindicações, subtende-se que tais produtos podem fazer mal à saúde do indivíduo. De toda forma, apesar da aparente contradição nos resultados dessas duas variáveis, pode-se afirmar que mais de $20 \%$ dos profissionais que estão cotidianamente, mais próximos dos usuários dos serviços das UBS, tanto pensam que plantas medicinais são inofensivas à saúde quanto que não existe contraindicações ao seu uso. Entre os que acham que existe contraindicações para a fitoterapia, a maior parte enxerga isso qualquer que seja a fase da vida do indivíduo (51\%), mas parcelas representativas dos entrevistados pensam que tais produtos estão contraindicados apenas na gravidez (14,3\%); na gravidez e durante a infância (10,2\%) e na gravidez, na infância e na velhice $(8,2 \%)$. Outras respostas menos frequentes foram: apenas na infância ( $\mathrm{n}=3,6,1 \%)$, somente entre idosos $(n=2,4,1 \%)$, entre gestantes e idosos $(n=2,4,1 \%)$ e, finalmente, ainda existe quem acredite que apenas idosos e crianças não devem usar tais produtos $(n=1,2 \%)$. Apresentamos todos esses percentuais, mesmo os de baixa frequência, a fim de ressaltar a diversidade de respostas e a fragilidade do conhecimento sobre fitoterapia racional, observada entre os profissionais de saúde que não possuem graduação. Reforçando o comentário do parágrafo anterior, esses resultados devem ser considerados pelos gestores municipais, quando da elaboração de políticas de aperfeiçoamento e atualização dos profissionais da APS.

Um último questionamento exclusivo para cada ACS e Técnico de Enfermagem, foi feito. Se alguma vez achou necessário comunicar ao médico ou enfermeiro da UBS que alguém fazia o uso de plantas medicinais ou fitoterápicos por conta própria. A maior parte desses profissionais ( $\mathrm{n}=42,66,7 \%)$ respondeu que "Não"; ou seja, muitos dos profissionais de saúde, que estão mais próximos do paciente, ainda não levam em consideração a importância clínica dos riscos de problemas ligados a medicamentos, inclusive fitoterápicos, tais como as possibilidades de interações medicamentosas prejudiciais. Talvez isso ocorra pelo mero desconhecimento de conceitos como o de farmacovigilância e do fato de que um acompanhamento mais especializado diminui os riscos de reações adversas e interações medicamentosas que podem acometer o usuário, que na maioria das vezes, é idoso e apresenta morbidades múltiplas.

Entre as associações de variáveis, as que mostraram significância estatística, foram: a variável gênero, com os profissionais que responderam o conceito adequado ou inadequado sobre fitoterapia. Percebeu-se que as mulheres, foram as que mais possuíram um conceito adequado sobre fitoterapia $(\mathrm{p}=0,027)$ (Tabela 4$)$. Esse fato talvez possa ser explicado pelo fato de que, culturalmente em nosso país, desde a mais tenra idade, as crianças são ensinadas que não somente o uso como também a preparação de chás e outros derivados de plantas medicinais, como qualquer atividade ligada à culinária, é uma tarefa mais "adequada" para as mulheres; apesar de que, nos últimos anos, tem se observado um aumento progressivo de interesse do público masculino por atividades antes consideradas como exclusivamente "femininas" (Pereira et al., 2021).

Tabela 4 - Associações estatísticas entre as variáveis "gênero" x "conceito adequado/inadequado sobre fitoterapia". Campina Grande, 2019.

\begin{tabular}{ccccc}
\hline & & Conceito Adequado & Conceito Inadequado & Total \\
\hline Sexo & $\mathrm{N}$ & 69 & 14 & 83 \\
Feminino & $(\%)$ & 83,13 & 16,87 & 100 \\
\hline Sexo & $\mathrm{n}$ & 05 & 05 & 10 \\
Masculino & $(\%)$ & 50 & 50 & 100 \\
\hline Total & $\mathrm{n}$ & 74 & 19 & 93 \\
& $(\%)$ & 79,57 & 20,43 & 100 \\
\hline
\end{tabular}

Teste Qui-quadrado (Teste Exato de Fisher) $=6.0268 ; \mathrm{p}=0.027$. Fonte: Autores (2021).

Outra associação significativa foi entre a variável "tipo de profissional”, recategorizada em nível médio/técnico e nível superior com a variável "prescrever e/ou orientar o uso de Plantas Medicinais e/ou Fitoterápicos", nos mostrou, como já era esperado, que os profissionais de nível superior prescrevem ou orientam o uso de plantas medicinais e fitoterápicos, mais que os 
profissionais de nível médio ( $\mathrm{p}=0,047$ ) (Tabela 5). Nesse sentido, cabe-nos ressaltar a importância estratégica dos profissionais de saúde, de nível superior, para a concretização dos princípios e diretrizes da Política Nacional de Plantas Medicinais e Fitoterápicos na Atenção Primária de Saúde, pois esses indivíduos não podem abdicar do seu papel de liderança nas equipes multiprofissionais do SUS.

Tabela 5 - Associações estatísticas entre as variáveis "tipo de profissional" x "prescrever e/ou orientar" o uso de Plantas Medicinais e/ou Fitoterápicos. Campina Grande, 2019.

\begin{tabular}{ccccc}
\hline & & Prescreve/orienta & Não prescreve/orienta & Total \\
\hline Nível & $\mathrm{N}$ & 26 & 37 & 63 \\
Médio/Técnico & $(\%)$ & 41,27 & 58,73 & 100 \\
\hline Nível Superior & $\mathrm{N}$ & 19 & 11 & 30 \\
& $(\%)$ & 63,33 & 36,67 & 100 \\
\hline Total & $\mathrm{N}$ & 45 & 48 & 93 \\
& $(\%)$ & 48,35 & 51,61 & 100 \\
\hline
\end{tabular}

Teste Qui-quadrado =3.9613; $\mathrm{p}=0.047$. Fonte: Autores (2021).

\section{Considerações Finais}

Diante da análise apresentada é possível afirmar que, apesar do uso de plantas medicinais e/ou fitoterápicos ser uma realidade entre boa parte dos pacientes da atenção primária em saúde na rede pública do município de Campina Grande, PB, a maioria dos profissionais entrevistados não prescreve e/ou orienta seus pacientes sobre o uso correto desses produtos, mesmo reconhecendo terem conhecimento que os usuários dos serviços de saúde, majoritariamente se automedicam com tais produtos. As principais justificativas apresentadas foram: a ausência da fitoterapia enquanto componente curricular na formação desses profissionais e a ausência de ações com vistas ao aperfeiçoamento e/ou atualização dos profissionais sobre eficácia e segurança do uso terapêutico de plantas medicinais e derivados.

Nesse sentido, a elaboração de ações estratégicas de educação em saúde, com o intuito de promover o uso seguro e eficaz de fitoterápicos, se torna necessária, tanto para os usuários, uma vez que as plantas medicinais e derivados são acessíveis e bem aceitos pela população, quanto para os profissionais, principalmente no que diz respeito ao incentivo e adesão dessa prática na Atenção Primária. Somente assim, mobilizando os protagonistas dos serviços ofertados nas UBS para a temática da fitoterapia e suas diversas potencialidades na otimização da assistência em saúde, é que obteremos um maior engajamento com vistas à implantação dos princípios e diretrizes da Política Nacional de Plantas Medicinais e Fitoterápicos e inserção da fitoterapia na APS do SUS.

\section{Referências}

Adom, M. B., Taher, M., Mutalabisin, M. F., Amri, M. S., Abdul Kudos, M. B., Wan Sulaiman, M. W. A., Sengupta, P., \& Susanti, D. (2017). Chemical constituents and medical benefits of Plantago major. Biomed Pharmacother, 96, 348-360.

Ali, M. M., Hashim, N., Aziz, A. S., \& Lasekan, O. (2020). Abacaxi (Ananas comosus): uma revisão abrangente dos valores nutricionais, compostos voláteis, benefícios para a saúde e potenciais produtos alimentares. Food Res Int, 137.

Almeida, M. S. M. (2018). Moringa oleifera Lam., seus benefícios medicinais, nutricionais e avaliação de toxicidade [Dissertação do upgrade ao Mestrado Integrado em Ciências Farmacêuticas, Faculdade de Farmácia da Universidade de Coimbra].

Araújo, A. K. L., Araújo-Filho, A. C. A., Ibiapina, L. G., Nery, I. S., Rocha, S. S. (2015). Dificuldades enfrentadas por enfermeiros na aplicabilidade da fitoterapia na atenção básica: uma revisão integrativa. Journal of Research: Fundamental Care Online, 7 (3), 2826-2834.

Araújo, C. R. F., Silva, A. B., Tavares, E. C., Costa, E. P., \& Mariz, S. R. (2014). Perfil e prevalência de uso de plantas medicinais em uma unidade básica de saúde da família em Campina Grande, Paraíba, Brasil. Revista de Ciências Farmacêuticas Básica e Aplicada, 35 (2), $233-238$.

Araujo, D. C. S. (2020). Efeito farmacológico de plantas do gênero Kalanchoe SPP.: revisão literária. http://rdu.unicesumar.edu.br/handle/123456789/7523. 
Azimi, M., \& Zahedi, M. J. (2021). Persian Herbal Medicine in Functional Dyspepsia: A Systematic Review. Current Drug Discovery Technologies, 18 (2), $272-281$.

Barnes, L. A., Leach, M., Anheyer, D., Brown, D., Carè, J., Lauche, R., Medina, D. N., Pinder, T. A., Bugarcic, A., \& Steel, A. (2020). The effects of Hedera helix on viral respiratory infections in humans: A rapid review. Adv Integr Med,7 (4), 222-226.

Barros, B. R. S. (2018). Caracterização estrutural e fitoquímica e investigação das Atividades antioxidante, antimicrobiana, citotóxica e imunomoduladora promovidas pelo extrato salino das folhas de Malpighia emarginata DC (acerola) [trabalho de conclusão de Curso -Licenciatura em Ciências Biológicas, Universidade Federal Rural de Pernambuco].

Bezerra, A. S. C. E., Franco, S. P. B., Mousinho, K. C., Fonseca, S. A., Matos-Rocha, T. J., Pavão, J. M. S. J., \& Santos, A. F. (2021). Diagnóstico situacional de profissionais de unidades de saúde da família em fitoterapia. Jornal Brasileiro de Biologia, 81 (3), 551-556.

Braga, J. C. B., \& Silva, L. R. Consumo de plantas medicinais e fitoterápicos no Brasil: perfil de consumidores e sua relação com a pandemia de COVID-19 (2021). Brazilian Journal of Health Review. 4 (1), 3831 - 3839.

Brasil (2016). Agência Nacional de Vigilância Sanitária. Memento de Fitoterápicos da Farmacopeia Brasileira (MFFB).115p.

Brasil (2016). Ministério da Saúde. Secretaria de Ciência, Tecnologia e Insumos Estratégicos. Departamento de Assistência Farmacêutica. Política e Programa Nacional de Plantas Medicinais e Fitoterápicos. 190 p.

Brasil (2020). Ministério da Saúde. Secretaria de Ciência, Tecnologia, Inovação e Insumos Estratégicos em Saúde. Departamento de Assistência Farmacêutica e Insumos Estratégicos. Relação Nacional de Medicamentos Essenciais: Rename 2020. 217p. http://conitec.gov.br/images/Rename-2020-final.pdf.

Caputo, L., Nazzaro, F., Souza, L. F., Aliberti, L., De Martino, L., Fratianni, F., Coppola, R., \& De Feo, V. (2017). Laurus nobilis: Composition of Essential Oil and Its Biological Activities. Molecules, 22 (6), 930.

Chandra, S., Rawat, D., Chandra, D., \& Rastogi, J. (2016). Nativity, phytochemistry, ethnobotany and pharmacology of Dianthus caryophyllus. Research Journal of Medicinal Plant, 10 (1), 1-9.

Cunha, J. M., Viana, E. S. M., Souza, J. T., \& Silva, S. S. (2016). Os efeitos do Hibisco (hibiscos sabdariffa) no emagrecimento. Revista Científica Univiçosa, $8(1), 657-661$.

Dhakad, A. K., Pandey, V. V., Beg, S., Rawat, J. M., \& Singh, A. (2018). Biological, medicinal and toxicological significance of Eucalyptus leaf essential oil: a review. J Sci Food Agric, 98 (3), 833-848.

Dosoky, N. S., \& Setzer, W. N. (2018). Biological Activities and Safety of Citrus spp. Essential Oils. Int J Mol Sci., 19 (7).

Feitosa, M. H. A. (2016). Inserção do Conteúdo Fitoterapia em Cursos da Área de Saúde. Revista Brasileira de Educação Médica, 40 (2), $197-203$.

Flor, A. S. S. O., \& Barbosa, W. L. R. (2015). Sabedoria popular no uso de plantas medicinais pelos moradores do bairro do sossego no distrito de Marudá PA. Rev. Bras. Pl. Med., 17 (4), 757-768.

Ge, S., Duo, L., Wang, J., Zhula, G., Yang, J., Li, Z., \& Tu, Y. (2021). A unique understanding of traditional medicine of pomegranate, Punica granatum L. and its current research status. Journal of Ethnopharmacology, 271.

Gomes, D. C., Coriolano, M. C., Holanda, V. N., \& Correia, M. T. S. (2018). Utilização de Achyrocline Satureioides (Lam) D.C na medicina popular e aplicações biológicas relatadas. Revista Interfaces: saúde, humanas e tecnologia, 6 (17),173-177.

Gontijo, M. B. A., \& Nunes, M. F. (2017). Práticas Integrativas e Complementares: Conhecimento e credibilidade de profissionais do serviço público de saúde. Trab. Educ. Saúde, 15 (1), 301-320.

Hanif, M., Mehmood, M. H., Ishrat, G., Virji, S. N., Malik, A., Ahmed, M., \& Gilani, A. H. (2019). Pharmacological basis for the medicinal use of Alcea rosea in airways disorders and chemical characterization of its fixed oils through GC-MS. Pak J Pharm Sci., 32 (5), 2347-2355.

He, X., Luan, F., Yang, Y., Wang, Z., Zhao, Z., Fang, J., Wang, M., Zuo, M., \& Li, Y. (2020). Passiflora edulis: An Insight Into Current Researches on Phytochemistry and Pharmacology. Front Pharmacol., 11 (617).

Heshmati, J., Morvaridzadeh, M., Sepidarkish, M., Fazelian, S., Rahimlou, M., Omidi, A., Palmowski, A., Asadi, A., \& Shidfar, F. (2020). Effects of Melissa officinalis (Lemon Balm) on cardio-metabolic outcomes: A systematic review and meta-analysis. Phytother Res., 34 (12), 3113-3123.

Jahurul, M. H. A., Patricia, M., Shihabul, A., Norazlina, M. R., Ramlah George, M. R., Noorakmar, A. W., Lee, J. S., Jumardi, R., Jinap, S., \& Zaidul, I. S. M. (2021). Uma revisão sobre as propriedades funcionais e nutricionais das sementes de noni (Morinda citrifolia L.) e seu óleo. Food Bioscience, 41.

Jia, S., Shen, M., Zhang, F., \& Xie, J. (2017). Recent Advances in Momordica charantia: Functional Components and Biological Activities. Int J Mol Sci., 18 (12), 2555.

Kothari, D., Lee, W. D., \& Kim, S. K. (2020). Allium Flavonols: Health Benefits, Molecular Targets, and Bioavailability. Antioxidants (Basel), 9 (9), 888.

Ladeiras, D. (2014). Estudo de compostos bioactivos e actividades biológicas do alecrim - Rosmarinus officinalis L. [Dissertação de Mestrado, Universidade Lusófona de Humanidades e Tecnologias. Escola de Ciências e Tecnologias da Saúde].

Lopes, M. A., Nogueira, I. S., Obici, S., \& Albiero, A. L. M. (2015). Estudo das plantas medicinais, utilizadas pelos pacientes atendidos no programa "Estratégia saúde da família" em Maringá/PR/Brasil. Rev. Bras. Pl. Med., 17 (4), 702-706.

Mariz, S. R., Juvino, E. O. R. S., Silva, A. O., Andrade, E. T. S., \& Dantas, L. Q. (2020). O uso de plantas medicinais entre pacientes da Atenção Primária. International Journal of Development Research, 10, 38034-38039. 
Marconi, M. A., \& Lakatos, E. M. (2017). Fundamentos de metodologia científica. Atlas, 346p.

Mattos, G., Camargo, A., Sousa, C. A., \& Zeni, A. L. B. (2018). Plantas medicinais e fitoterápicos na Atenção Primária em Saúde: percepção dos profissionais. Ciência \& Saúde Coletiva, 23 (11), 3735-3744.

Medeiros, I. C. (2018). Avaliação do conhecimento e uso de plantas medicinais e fitoterápicos por dentistas do Seridó Potiguar [Monografia de Especialização, Curso de Residência Multiprofissional em Atenção Básica, Escola Multicampi de Ciências Médica, Universidade Federal do Rio Grande do Norte].

Miranda, S. S., Gandolfo, J. L., Vieira, R. G. C., Zanatta, M. C. A., Alves, J. R.F., Almeida, C. C. S., \& Faria, T. V. (2020). O chá da folha de Morus nigra como agente promotor de qualidade de vida em mulheres na transição menopáusica. Revista Eletrônica Acervo Saúde, 12 (9), e4288.

Motta, A. O., Lima, L. C. S., \& Vale, C. R. (2016). Levantamento do uso das plantas medicinais em um centro de educação infantil em Goiânia- GO. Revista da Universidade Vale do Rio Verde, 14 (1), 629 - 646.

Nascimento-Júnior, B. J., Tínel, L. O., Silva, E. S., Rodrigues, L. A., Freitas, T. O. N., Nunes, X. P., \& Amorim, E. L. C. (2016). Avaliação do conhecimento e percepção dos profissionais da estratégia de saúde da família sobre o uso de plantas medicinais e fitoterapia em Petrolina-PE, Brasil. Rev. Bras. Pl. Med., 18 (1), $57-66$.

Pereira, M. G., Silva, B. N., Vieira, L. R. F., Quaresma, F. E. L., Cesário, P. F. O., \& Silva, C. R. D. V. (2021). Saúde mental de mulheres profissionais de saúde no Brasil: uma revisão narrativa. In: Nascimento ARS, Rodrigues ARGM, Castro AP e Medeiros NM. Saúde mental e suas interações: rompendo paradigmas (pp. 24-29). https://www.poisson.com.br/livros/individuais/Saude_Mental_Paradigmas/Saude_Mental_Paradigmas.pdf\#page=24.

Piriz, M. A., Lima, C. A. B., Jardim, V. M. R., Mesquita, M. K., Souza, A. D. Z., \& Heck, R. M. (2014). Plantas medicinais no processo de cicatrização de feridas: uma revisão de literatura. Rev. Bras. Pl. Med., 16 (3).

Prakash, A., \& Baskaran, R. (2018). Acerola, an untapped functional superfruit: a review on latest frontiers. J Food Sci Technol., 55 (9), $3373-3384$.

El mokni, R., Youssef, F. S., Jmii, H., Khmiri, A., Bouazzi, S., Jlassi, I., Jaidane, H., Dhaouadi, H., Ashour, M. L., \& Hammami, S. (2019). O óleo essencial de tunisinas ambrosioides Dysphania e sua antimicrobiana e propriedades antivirais. Journal of Essential Oil Bearing Plants, 22 (1), $282-294$.

Santos, M. S., Amarello, M. M., Vigeta, S. M. G., Horta, A. L. M., Tanaka, L. H., \& Souza, K. M. J. (2018). Práticas integrativas e complementares: Avanços e desafios para a promoção da saúde de idosos. Revista Mineira de Enfermagem., 22 (1126).

Silva, A. F. L., \& Barros, L. A. A. (2021). Evaluation of practices for the use of medicinal plants in the City of Caxias-MA. Research, Society and Development, $10(4)$.

Silva, E. L. P., Soares, J. C. F., Machado, M. J., Reis, I. M. A., \& Cova, S. C. (2020). Avaliação do perfil de produção de fitoterápicos para o tratamento de ansiedade e depressão pelas indústrias farmacêuticas brasileiras. Braz. J. of Develop., 6 (1), 3119-3135.

Silva, H. G. N., et al (2018). Retrato sociocultural: o uso de plantas medicinais por pacientes idosos com diabetes melittus tipo 2 . Revista Interdisciplinar, 11 (4), 21-29.

Silva, M. D. P., Marini, F. S., \& Melo, R. S. (2015). Levantamento de plantas medicinais cultivadas no município de Solânea, agreste paraibano: reconhecimento e valorização do saber tradicional. Rev. Bras. Pl. Med. Campinas, 17 (4), 881-890.

Silva, P. A. M., Oliveira, A. E. F., Souza, B. E., Barbosa, C. T., Oliveira, L. S., Pereira, A. S., \& Horta, N. C. (2021). Práticas integrativas e complementares em saúde: possibilidades para o cuidado integral. Revista Eletrônica Acervo Saúde, 13 (1).

Singh, N., Rao, A. S., Nandal, A., Kumarb, S., Yadav, S. S., Ganaie, A. S., \& Narasimhan, B. (2021). Phytochemical and pharmacological review of Cinnamomum verum J. Presl - a versatile spice used in food and nutrition. Food Chemistry., 338 (15).

Souza, A. D. Z., Heinen, H. M., Amestoy, S. C., Mendieta, M. C., Piriz, M. A., \& Heck, R. M. (2016). O Processo de trabalho dos enfermeiros da atenção primária e a Política Nacional de Plantas Medicinais/Fitoterápicos. Rev. Bras. Pl. Med., 18 (2), 480-487.

Subramaniam, G., Sivasamugham, L. A., \& Yew, X. Y. (2020). Antibacterial activity of Cymbopogon citratus against clinically important bactéria. South African Journal of Chemical Engineering. 34 (1).

Tabeshpour, J., Razavi, B. M., \& Hosseinzadeh, H. (2017). Effects of Avocado (Persea americana) on Metabolic Syndrome: A Comprehensive Systematic Review. Phytother Res., 31 (6), 819-837.

Tafazoli, A., \& Moghadam, E. T. (2020). Camellia Sinensis Mouthwashes in Oral Care: a Systematic Review. J Dent (Shiraz)., 21 (4), $249-262$.

Tomazzoni, M. I. (2004). Subsídios para a introdução do uso de fitoterápicos na rede básica de saúde do município de Cascavel/PR [Dissertação de Mestrado, Programa de Pós- Graduação em Enfermagem, Universidade Federal do Paraná].

Valverde, M. E., Orona-Tamayo, D., Nieto-Rendón, B., Paredes-López, O. (2017). Antioxidant and Antihypertensive Potential of Protein Fractions from Flour and Milk Substitutes from Canary Seeds (Phalaris canariensis L.). Plant Foods Hum Nutr., 72 (1), $20-25$.

Vargas, E. C. A. (2017). Interface entre os saberes populares e científicos sobre plantas medicinais: perspectiva da autonomia do cuidado em saúde [Dissertação de Mestrado Profissional em Enfermagem Assistencial, Universidade Federal Fluminense].

Vieira, E. F., Pinho, O., Ferreira, I. M. P. L. V. O., \& Delerue-Matos, C. (2019). Chayote (Sechium edule): A review of nutritional composition, bioactivities and potential applications. Food Chem., 275, 557-568.

Virgínio, T. B., Castro, K. S., Lima, A. L. A., Rocha, J. V., Bonfim, I. M., \& Campos, A. R. (2018). Utilização de plantas medicinais por pacientes hipertensos e diabéticos: estudo transversal no nordeste brasileiro. Revista Brasileira em Promoção da Saúde, 31 (4). 
Research, Society and Development, v. 10, n. 5, e29310514928, 2021

(CC BY 4.0) | ISSN 2525-3409 | DOI: http://dx.doi.org/10.33448/rsd-v10i5.14928

Zeljkovi'c, S. C., Šišková, J., Komzáková, K., De Diego, N., Kaffková, K., \& Tarkowski, P. (2021). Phenolic Compounds and Biological Activity of Selected Mentha Species. Plants, 10 (550).

Zeni, A. L. B., Parisotto, A. V., Mattos, G., \& Helena, E. T. S. (2017). Utilização de plantas medicinais como remédio caseiro na Atenção Primária em Blumenau, Santa Catarina, Brasil. Ciência \& Saúde Coletiva, 22 (8), 2703-2712. 